\title{
Baixos níveis de aptidão física relacionada à saúde em universitários
}

\section{Resumo}

Este estudo teve por objetivo identificar a prevalência de baixos níveis de aptidão física relacionada à saúde em universitários, quanto aos componentes músculo-esqueléticos (motores). Participaram da amostra 234 estudantes (112 moças e 122 rapazes, entre 17 e 29 anos), matriculados em diversos cursos da Universidade Federal de Santa Catarina. Foi utilizada uma bateria de testes proposta pela Sociedade Canadense de Fisiologia do Exercício. Os testes propostos foram: força de preensão manual; extensão dos braços; flexão do tronco; impulsão vertical; resistência abdominal e força dorsal. Os resultados apontam uma prevalência de aptidão física abaixo dos indicadores recomendados à saúde de 16,6\% (13,4\% feminino e 19,6\% masculino). Especificamente, observaram-se os seguintes índices de prevalência de universitários que não atingiram os critérios recomendados: $69,2 \%$ para a potência de membros inferiores, $62 \%$ para extensão de braços, $38,4 \%$ para a preensão manual, $13,2 \%$ para flexão de tronco, $11,1 \%$ para força dorsal e 10,7\% para força abdominal. Em se tratando de individuos jovens, observou-se que boa parte dos universitários encontra-se com baixos niveis de aptidão física, sugerindo a importância da implantação de programas para o desenvolvimento e/ou manutenção da aptidão física destes estudantes, fornecendo subsídios para que estes adotem um estilo de vida mais ativo e saudável por toda a sua vida.

UnItermos: Aptidão Física; Saúde; Jovens.

\section{Introdução}

A atividade física, a aptidão física e a saúde relacionam-se entre si de forma positiva e linear, assim, pessoas fisicamente ativas apresentam melhores níveis de aptidão e saúde que seus pares pouco ativos ou sedentários (Malina, 2001; Shephard \& Bouchard, 1994).

São duas as principais abordagens ligadas ao conceito de aptidão física: uma relacionada ao desempenho esportivo e outra à saúde. Em decorrência do aumento da expectativa de vida das populaçôes, tem havido um maior interesse sobre essa última em detrimento da esportiva, motivado pelo reconhecimento crescente do papel da atividade física regular na promoção e na manutenção da saúde (AMERICAN COLEGE OF SPORTS Medicine - ACSM, 1998; Caspersen, Powell \& Christensen, 1985; Paffenbarger, 1994).

Assim, no campo da saúde, a aptidão física está relacionada com a capacidade do indivíduo de realizar atividades do cotidiano com vigor e energia e demonstrar menor risco de desenvolver doenças ou condições crônico-degenerativas associadas a baixos níveis de atividade física (ACSM, 1998). Existe um número cada vez maior de evidências que comprovam os benefícios da aptidão física para a saúde (BLAIR, KoHL, Paffenbarger, Gibbons \& Macera, 1995; Canadian Society For EXercise Physiology - CSEP, 2004; LeE \& Paffenbarger Junior, 2000; Zoeller, 2007).

Porém, observa-se que em decorrência dos inúmeros avanços tecnológicos, o estilo de vida contemporâneo cada vez mais induz as pessoas à inatividade física e à adoção de hábitos de vida inadequados (Blair, Norton \& Leon, 1996; U.S.Department of Health and Human Services - HHS, 2007).

Os reflexos deste estilo de vida aparecem cada vez mais cedo na população, fazendo com que vários estudos evidenciem o aumento de taxas de prevalência de diversas doenças como hipertensão arterial, diabetes tipo 2 e outros problemas cardiovasculares, bem como de sobrepeso e obesidade em idades cada vez mais precoces (FISBERG, 2006; Lima, 2004; Myers, 2003; SARTORELli \& Franco, 2003). 
Em decorrência destas evidências, a Federação Internacional de Medicina Esportiva e a Organização Mundial de Saúde têm estimulado a população em geral, a envolver-se em atividades físicas regulares (INTERNATIONAL FEDERATION OF SPORTS Medicine/World Health Organization - FIMS/ WHO, 1998). O combate ao sedentarismo tem sido estimulado, baseando-se em pesquisas

\section{Procedimentos metodológicos}

\section{Caracterização do estudo}

Trata-se de estudo transversal realizado com universitários matriculados na Educação Física Curricular (oferecida como disciplina optativa para os alunos de 70 cursos, considerando turno e habilitaçōes) da Universidade Federal de Santa Catarina, Brasil. Primeiramente, todos os professores foram convidados a colocar as suas turmas a disposição da pesquisa e liberar os seus respectivos horários para a coleta dos dados. Em seguida, os alunos foram informados sobre os objetivos do estudo e as datas das coletas, bem como sobre os procedimentos metodológicos.

\section{Amostra}

Conforme informações da secretaria acadêmica estavam matriculados 1.034 alunos. Participaram do estudo, 234 (22,6\%) universitários, sendo 122 do sexo masculino e 112 do sexo feminino.

\section{Comitê de ética}

A presente pesquisa foi aprovada pelo Comitê de Ética em Pesquisa da Universidade Federal de Santa Catarina (Protocolo no 096/2007). Os dados foram coletados após a assinatura do consentimento livre e esclarecido pelos universitários, sendo-lhes assegurado o sigilo sobre as informaçôes individuais.

\section{Coleta de dados}

A coleta dos dados foi realizada por avaliadores experientes, no início do semestre letivo, e foram seguidas as padronizaçóes preconizadas pelo Plano Canadense de Avaliação da Atividade Física, Aptidão e Estilo de Vida (CSEP, 2004). epidemiológicas que associam uma menor morbidade e mortalidade em indivíduos fisicamente ativos de todas as idades (LeE \& PAfFenbarger Junior, 2000).

Desta forma, a presente pesquisa buscou verificar a prevalência de baixos níveis de aptidão física relacionada à saúde (AFRS) quanto aos componentes músculo-esqueléticos (motores) de universitários.

\section{Medidas antropométricas}

Foram mensuradas as seguintes medidas antropométricas: massa corporal, estatura, cinco dobras cutâneas (bíceps, tríceps, subescapular, supra-ilíaca e panturrilha medial), e o perímetro do abdômen.

\section{Aptidão músculo-esquelética}

Para a aptidão física foram incluídos seis testes motores - Força de Preensão Manual, Extensão de Braços, Flexão do Tronco (sentar-e-alcançar), Força abdominal, Impulsão vertical e Força dorsal.

\section{Classificação da aptidão física}

Para a classificação geral da AFRS dos universitários foram utilizadas as normas da CSEP (2004), que considera idade e sexo, as quais permitem a interpretação da aptidão física, individualmente ou em conjunto, a partir de pontos de corte que definem categorias de benefícios à saúde. A AFRS dos indivíduos foi então classificada em cinco categorias: "Excelente", "Muito Boa", "Boa", "Regular" e "Necessita Melhorar". A condição de "Baixo nível de aptidão física" foi definida agrupando-se as categorias: "Regular" e "Necessita Melhorar", pois segundo a CSEP (2004), a aptidão física nestas categorias geralmente está associada a riscos para saúde.

\section{Análise estatística}

Os dados foram analisados por estatística descritiva (média, desvio padrão e frequência relativa). As diferenças de prevalência entre os sexos foram obtidas através do teste "t" proporçōes. O cálculo dos dados foi feito através do programa MedCalc, utilizando-se um nível de significância $\mathrm{p}<0,05$. 


\section{Resultados}

Os valores médios das características de idade, estatura e massa corporal dos univeristários são apresentados na TABELA 1.

TABELA 1 - Características gerais de idade, estatura e massa corporal dos universitários, por sexo.

\begin{tabular}{lcccc}
\hline & \multicolumn{2}{c}{ Masculino } & \multicolumn{2}{c}{ Feminino } \\
\cline { 2 - 5 } & Média & DP & Média & DP \\
\hline Idade (anos) & 22,7 & 3,8 & 23,3 & 6,6 \\
Estatura (cm) & 176,0 & 0,1 & 164,0 & 0,1 \\
Massa corporal (kg) & 70,8 & 11,3 & 57,4 & 9,8 \\
\hline
\end{tabular}

A TABELA 2 apresenta a proporção de universitários com baixa aptidão em cada variável da aptidão física relacionada à saúde quanto aos componentes motores.

A prevalência geral indicou que $16,6 \%$ dos universitários apresentaram aptidão física abaixo dos indicadores recomendados à saúde para os componentes músculo-esqueléticos (motores), sendo 13,4\%, do sexo feminino, e 19,7\%, do masculino.

Foram encontradas diferenças estatísticas significativas nos testes de extensão de braços e força/resistência abdominal, entre homens e mulheres ( $p<0,05)$; nestas variáveis observou-se que o sexo feminino apresentou maior prevalência de baixa aptidão física em relação ao masculino; $70,5 \%$ e $16,1 \%$ para mulheres e $54,1 \%$ e $6,8 \%$ para homens, respectivamente $(\mathrm{p}<0,05)$. Nas demais variáveis, quando comparadas entre os sexos, não foram evidenciadas diferenças estatísticas significativas.

TABELA 2 - Proporções de universitários com baixa aptidão em cada variável da Aptidão Física relacionada à Saúde.

\begin{tabular}{|c|c|c|c|c|}
\hline Variável & Masculino \% & Feminino \% & Geral \% & p valor \\
\hline Força de preensão manual & 36,0 & 41,1 & 38,4 & 0,515 \\
\hline Extensão de braços & 54,1 & 70,5 & 62,0 & $0,014^{*}$ \\
\hline Flexão de tronco & 12,3 & 14,3 & 13,2 & 0,796 \\
\hline Impulsão vertical & 69,6 & 68,8 & 69,2 & 0,993 \\
\hline Força/Resistência abdominal & 6,8 & 16,1 & 10,7 & $0,041^{*}$ \\
\hline Força dorsal & 14,8 & 7,2 & 11,1 & 0,101 \\
\hline Baixo nível de aptidão física geral & 19,7 & 13,4 & 16,6 & 0,264 \\
\hline
\end{tabular}

\section{Discussão}

O objetivo deste estudo foi verificar a prevalência de baixos níveis de aptidão física relacionada à saúde quanto aos componentes músculo-esqueléticos de universitários da Universidade Federal de Santa Catarina, Brasil. Com base nos resultados observou-se prevalência de 16,6\% dos indivíduos apresentaram baixos níveis de aptidão; mesmo que esta proporção não seja alta, deve-se dar uma atenção especial a este fato serem indivíduos jovens. Nesta pesquisa não foram investigados os motivos da baixa aptidão física, porém, acredita-se que podem estar associados a essa variável, os hábitos sedentários cada vez mais prevalentes entre adultos jovens (HALLAL, VICTORA, Wells \& Lima, 2003). A literatura relata que a participação em atividades físicas declina consideravelmente com o aumento da idade, em especial da adolescência para o adulto (MALINA, 2001).
A atividade física regular é essencial para manter e melhorar a aptidão física, bem como aprimorar a saúde como um todo, diminuindo o risco do aparecimento de doenças e agravos crônicos que incluem as cardiovasculares, diabetes, osteoporose, obesidade, dislipidemias, depressão e alguns tipos de câncer, bem como diminui o risco de afecçôes osteomusculares (CSEP, 2004; LiCHTENSTEIN, APPEL, Brands, Carnethon, Daniels, Franch, Franklin, Kris-Etherton, Harris, Howard, Karanja, LefeVre, Rudel, Sacks, Van Horn, Winston \& Wylie-Rosett, 2006; Pate, 1983; Zoeller, 2007), baseando-se nestas afirmaçōes, bons níveis de aptidão física podem trazer benefícios para a saúde das pessoas contribuindo também para uma melhor qualidade de vida. 
É durante a juventude que há ganho de hábitos duradouros de atividade física que vão até a velhice, assim parece razoável estimular mudanças de comportamento nestas idades, para que estes indivíduos mantenham esse hábito por toda a vida (Lopes, Maia, Oliveira, SEAbra \& Garganta, 2003; Souza \& Duarte, 2005). Em se tratando de saúde e qualidade de vida, bons níveis de aptidão física auxiliam no desenvolvimento das tarefas diárias (CASPERSEn, POWEll \& Christensen, 1985) diminuem o risco de doenças, reduzem os níveis de estresse e depressão e melhoram a auto-estima (CSEP, 2004); bem como seus componentes abrigam atributos biológicos que oferecem alguma proteção ao aparecimento e ao desenvolvimento de distúrbios orgânicos, induzidos pelo comprometimento da condição funcional (Pate, 1983; Yancey, Fielding, Flores, Sallis, McCarthy \& Breslow, 2007).

Níveis adequados de força muscular são importantes para um bom funcionamento músculoesquelético e contribuem para preservação da musculatura bem como das articulações ao longo da vida. Por outro lado, baixos níveis desta variável, vão gradativamente dificultando a realização de diversas tarefas cotidianas (GLANER, 2003) e na condição de estudante, acabam gerando problemas relacionados ao trabalho e estudos destes universitários.

A literatura relata que baixos níveis deste componente agravam a incidência de distúrbios músculoesqueléticos, e estão diretamente associados a presença de doenças que incapacitam milhares de trabalhadores e estudantes; as lombalgias representam uma das principais causas de incapacidade temporária e aposentadoria precoce, tornando-se um importante problema de saúde pública (CECIN, 2001).

Quando comparadas às prevalências de baixa aptidão física entre os sexos, foram observadas diferenças estatisticamente significativas nas variáveis de extensão de braços e força/resistência abdominal. Nestas variáveis, o sexo masculino apresentou menor proporção de indivíduos classificados com aptidão física insatisfatória em relação ao sexo feminino, resultados similares também foram relatados na litetatura (Loch, Konrad, SANTOS \& NAHAS, 2006), porém, divergentes daqueles encontrados por BIM e NARDO JÚNIOR (2005).

A flexibilidade (flexão do tronco) e a força dorsal, foram as variáveis com menor prevalência de baixa aptidão física, indicando que a maioria dos indivíduos atingiu índices sem riscos à saúde. Uma boa flexibilidade, aliado a um bom desenvolvimento músculo-esquelético, em especial na região lombar e abdominal, contribuem para uma correta postura corporal, oferecendo certa proteção à coluna lombar (Toscano \& Egypto, 2001).

Neste estudo, observou-se que as maiores prevalências de baixa aptidão foram encontradas nos testes que representam componentes da força muscular, como de preensão manual, extensão de braços e impulsão vertical; o que indica que estes universitários apresentam níveis de força muscular abaixo do esperado, resultados esses que vão ao encontro da literatura (Pereira \& GRAUP, 2007).

A principal limitação do presente estudo, referese ao viés de seleção, já que a amostra investigada não representa a totalidade dos alunos da UFSC, mas apenas a parcela de estudantes que freqüentava as aulas de Educação Física Curricular. Outra limitação, refere-se ao fato de não ter sido avaliada a componente cardiorespirátioria, haja vista que a mesma é relevante no diagnóstico da aptidão física. Porém, apesar destas limitaçóes, esta amostra possui uma boa representatividade pelo fato de os acadêmicos matriculados na Educação Física Curricular, proceder dos diversos cursos da instituição. Assim, supõe-se, que os universitários aqui avaliados, apresentam similar padrão de aptidão física que seus pares, haja vista que a mensuração da aptidão física ocorreu no início do semestre letivo. Como ponto positivo, os achados do presente estudo reforçam a necessidade de se adotar estratégias que visem à promoção da saúde no campus universitário.

Sendo a "internet" uma ferramenta utilizada com frequência por estudantes universitários, a mesma poderia ser utilizada como meio de informação e de intervenção. As evidências do potencial da "web" para informar e facilitar as mudanças de comportamento em relação à $\mathrm{AF}$, têm sido recentemente relatadas na literatura (Ferney \& Marhall, 2006; SpitTAels, Bourdeaudhuij \& Vandelanotte, 2007).

A análise da aptidão física geral dos universitários sugere que prevalência de baixa aptidão física, requer especial atenção, principalmente por se tratar de adultos jovens. Segundo a metodologia utilizada no presente estudo, esta situação indica que a AFRS dos universitários se situa dentro de um intervalo de resultados geralmente associados a riscos para a saúde. A passagem desta categoria para a "Boa" exige a solicitação de grandes massas musculares contra uma resistência de forma vigorosa, duas ou três vezes por semana (CSEP, 2004). Esta é uma etapa muito importante para aumentar os benéficos-saúde associados à aptidão músculo-esquelética. 


\begin{abstract}
Low levels of health-related physical fitness among university students

The aim of this study was to identify the prevalence of low health-related physical fitness levels among university students in terms of muscle-skeletal components (motor performance). A total of 234 students (112 girls and 122 boys aged 17 to 29 years), enrolled in different courses of the Federal University of Santa Catarina, Brazil, participated in the study. The test battery proposed by the Canadian Society for Exercise Physiology was used: handgrip strength, push-ups, sit and reach, vertical jump, partial curl-ups, and back extension. The results showed a prevalence of health-related physical fitness indicators below the recommended $(16.6 \%, 13.4 \%$ for girls and $19.6 \%$ for boys). Specifically, the following prevalence rates of university students who did not reach the recommended criteria were observed: $69.2 \%$ for vertical jump, 62\% for arm extension, $38.4 \%$ for handgrip strength, 13.2\% for trunk flexibility, 11.1\% for dorsal strength, and 10.7\% for abdominal endurance. Low physical fitness levels were observed among the evaluated university students, a finding suggesting the importance of the implantation of programs for the development and/or maintenance of physical fitness so that these students adopt a more active and healthier lifestyle throughout their lives.
\end{abstract}

UnITERMS: Physical fitness; Health; University students.

\title{
Referências
}

AMERICAN COLLEGE OF SPORTS MEDICINE (ACSM). American College of Sports Medicine position stand: the recommended quantity and quality of exercise for developing and maintaining cardiorespiratory and muscular fitness, and flexibility in healthy adults. Medicine \& Science in Sports \& Exercise, Madison, v.30, n.6, p.975-91, 1998.

BLAIR, S.N.; KOHL, H.W.; PAFFENBARGER, R.S.; CLARK, D.G.; COOPER, K.H.; GIBBONS, L.W. Physical fitness and all-cause mortality: a prospective study of healthy men and women. Journal of the American Medical Association, Chicago, v.262, n.17, p.2395-401, 1989.

BLAIR, S.N.; NORTON, E.; LEON, A.S. Physical activity, nutrition and chronic disease. Medicine $\&$ Science in Sports \& Exercise, Madison, v.28, n.3, p.335-49, 1996.

CECIN, H.A. (Coord./Ed.). Diagnóstico e tratamento das lombalgias e lombociatalgias. São Paulo: Associação Médica Brasileira/Conselho Federal de Medicina, 2001. (Projeto Diretrizes).

BIM, R.H.; NARDO JUNIOR, N. Aptidão física relacionada saúde de adolescentes estagiários da Universidade Estadual de Maringá. Revista Acta Scientiarum: Health Sciences, Maringá, v.27, n.1, p.77-85, 2005.

CANADIAN SOCIETY FOR EXERCISE PHYSIOLOGY (CSEP). The Canadian physical activity, fitness and lifestyle appraisal: CSEP's guide to health active living. Ottawa: CSEP, 2004.

CASPERSEN, C.J, POWELL, K.E.; CHRISTENSEN, G.M. Physical activity, exercise, and physical fitness: definitions and distinctions for health-relates research. Public Health Reports, Washington, v.100, n.2, p.172-9, 1985.

FERNEY, S.L.; MARSHALL, A.L. Website physical activity interventions: preferences of potential users. Health Education Research, Oxford, v.21, n.4, p.560-6, 2006.

INTERNATIONAL FEDERATION OF SPORTS MEDICINE. WORLD HEALTH ORGANIZATION (FIMS/ WHO). Committee on Sports and Children. Sports and children: consensus statement on organized sports for children. Bulletin of the World Health Organization, Geneva, v.76, n.5, p.445-7, 1998.

FISBERG, M. Obesidade na infância e adolescência. Revista Brasileira de Educação Física e Esporte, São Paulo, v.20, p.163-4, 2006. Suplemento n.5.

GLANER, M.F. Importância da aptidão física relacionada à saúde. Revista Brasileira de Cineantropometria e Desempenho Humano, Florianópolis, v.5, n.2, p.75-85, 2003.

HALLAL, P. C.; VICTORA, C.G.; WELLS, J. C. K.; LIMA. R. C. Physical inactivity: prevalence and associated variables in Brazilian adults. Medicine \& Science in Sports \& Exercise, Madison, v.35, n.11, p.1894-900, 2003.

LEE, M.; PAFFENBARGER JUNIOR, R.S. Associations of light, moderate and vigorous intensity physical activity with longevity. American Journal of Epidemiology, Baltimore, v.151, n.3, p.293-9, 2000. 
LICHTENSTEIN, A.H.; APPEL, L.J.; BRANDS, M.; CARNETHON, M.; DANIELS, S.; FRANCH, H.A.; FRANKLLIN, B.; KRIS-ETHERTON, P.; HARRIS, W.S.; HOWARD, B.; KARANJA, N.; LEFEVRE, M.; RUDEL, L.; VAN HORN, L.; WINSTON, M.; WYLIE-ROSETT, J. Diet and lifestyle recommendations revision 2006: a scientific statement from the American Heart Association Nutrition Committee. Circulation, Boston, v.114, p.82-96, 2006. LIMA, E.M. Avaliação dos fatores de risco associados com a elevação da pressão arterial em crianças e adolescentes. Jornal de Pediatria, Porto Alegre, v.80, n.1, p.3-5, 2004.

LOCH, M.R.; KONRAD, L.M.; SANTOS, P.D.; NAHAS, M.V. Perfil da aptidão física relacionada à saúde de universitários da educação física curricular. Revista Brasileira de Cineantropometria e Desempenho Humano, Florianópolis, v.8, n.1, p.64-71, 2006.

LOPES, V.P.; MAIA, J.A.R.; OLIVEIRA, M.M.C.; SEABRA, A.; GARGANTA, R. Caracterização da atividade física habitual em adolescentes de ambos os sexos através de acelerometria e pedometria. Revista Paulista de Educação Física, São Paulo, v.17, n.1, p.51-63, 2003.

MALINA, R.M. Physical activity and fitness: pathways from childhood to adulthood. American Journal of Human Biology, Cambridge, v.13, n.2, p.162-72, 2001.

MYERS, J. Exercise and cardiovascular health. Circulation, Boston, v.107, p.2-5, 2003.

PAFFENBARGER JUNIOR, R.S. Forty years of progress: physical activity, health, and fitness. In: AMERICAN College of Sports Medicine 40th Anniversary Lectures. Madison: ACSM, 1994.

PATE, R.R. A new definition of youth fitness. The Physician and SportsMedicine, Berwyn, v.11, n.4, p.77-83, 1983. PEREIRA, E.F; GRAUP, S. Aptidão física relacionada à saúde e ao desempenho atlético de calouros de Educação física. Lecturas, Educación Fisica y Deportes, Revista Digital, Buenos Aires, v.11, n.104, 2007.

SARTORELLI, D.S, FRANCO, L.J. Tendências do diabetes mellitus no Brasil: o papel de transição nutricional. Cadernos de Saúde Pública, Rio de Janeiro, v. 19, p. 29-36, 2003. Suplemento 1.

SHEPHARD, R.J.; BOUCHARD, C. Principal components of fitness: relationship to physical activity and life-style. Canadian Journal of Applied Physiology, Champaign, v.19, p.200-14, 1994.

SOUZA, G.S.; DUARTE, M.F.S. Estágios de mudança de comportamento relacionados à atividade física em adolescentes. Revista Brasileira de Medicina do Esporte, São Paulo, v.11, n.2, p.104-8, 2005.

SPITTAELS, H.; BOURDEAUDHUIJ, I.; VANDELANOTTE, C. Evaluation of a website-delivered computer-tailored intervention for increasing physical activity in the general population. Preventive Medicine, New York, v.44, n.4, p.209-17, 2007. TOSCANO, J.J.O.; EGYPTO, E.P. A influência do sedentarismo na prevalência de lombalgia. Revista Brasileira de Medicina do Esporte, São Paulo, v.7, n.4, p.132-7, 2001

U.S. DEPARTMENT OF HEALTH AND HUMAN SERVICES (HHS). Centers for Disease Control and Prevention. Physical activity and good nutrition: essential elements to prevent chronic diseases and obesity, 2007. Disponível em: $<$ http://www.cdc.gov/nccdphp/publications/aag/pdf/dnpa.pdf>. Acesso em: abr. 2007.

ZOELLER, R.F. Physical activity and fitness in prevention of coronary heart disease and associated risk factors. American Journal of Lifestyle Medicine, Boston, v.29, n.1, p.29-33, 2007.

YANCEY, A.K.; FIELDING, J.E.; FLORES, G.R.; SALLIS, J.F.; McCARTHY, W.J.; BRESLOW, L. Creating a robust public health infrastructure for physical activity promotion. American Journal of Preventive Medicine, Boston, v.32, n.1, p.68-78, 2007.

\begin{tabular}{|c|c|}
\hline ENDEREÇO & \\
\hline Edio Luiz Petroski & \\
\hline Departamento de Educação Física & Recebido para publicação: 26/11/2007 \\
\hline Universidade Federal de Santa Catarina & 1a. Revisão: 04/10/2008 \\
\hline Campus Universitário - Trindade & 2a. Revisão: 10/o8/2009 \\
\hline $\begin{array}{r}\text { 88040-900 - Florianópolis - SC - BRASIL } \\
\text { e-mail: petroski@cds.ufsc.br }\end{array}$ & Aceito: 23/09/2009 \\
\hline
\end{tabular}

54 - Rev. bras. Educ. Fís. Esporte, São Paulo, v.24, n.1, p.49-54, jan./mar. 2010 